Idiopathic Pulmonary Fibrosis 

Hiroyuki Nakamura - Kazutetsu Aoshiba Editors

\section{Idiopathic Pulmonary Fibrosis}

Advances in Diagnostic Tools and Disease Management

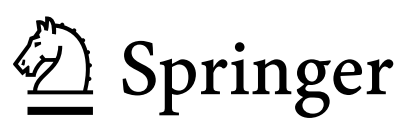




\author{
Editors \\ Hiroyuki Nakamura \\ Department of Respiratory Medicine \\ Tokyo Medical University \\ Ibaraki Medical Center \\ Ibaraki, Japan
}

\author{
Kazutetsu Aoshiba \\ Department of Respiratory Medicine \\ Tokyo Medical University \\ Ibaraki Medical Center \\ Ibaraki, Japan
}

\section{ISBN 978-4-431-55581-0 \\ ISBN 978-4-431-55582-7 (eBook) \\ DOI 10.1007/978-4-431-55582-7}

Library of Congress Control Number: 2015950193

\section{Springer Tokyo Heidelberg New York Dordrecht London \\ (C) Springer Japan 2016}

This work is subject to copyright. All rights are reserved by the Publisher, whether the whole or part of the material is concerned, specifically the rights of translation, reprinting, reuse of illustrations, recitation, broadcasting, reproduction on microfilms or in any other physical way, and transmission or information storage and retrieval, electronic adaptation, computer software, or by similar or dissimilar methodology now known or hereafter developed.

The use of general descriptive names, registered names, trademarks, service marks, etc. in this publication does not imply, even in the absence of a specific statement, that such names are exempt from the relevant protective laws and regulations and therefore free for general use.

The publisher, the authors and the editors are safe to assume that the advice and information in this book are believed to be true and accurate at the date of publication. Neither the publisher nor the authors or the editors give a warranty, express or implied, with respect to the material contained herein or for any errors or omissions that may have been made.

Printed on acid-free paper

Springer Japan KK is part of Springer Science+Business Media (www.springer.com) 


\section{Preface}

Owing to the remarkable advances in the fields of molecular biology and genomics/ genetics in recent years, medicine has progressed rapidly in various fields, including respiratory medicine. Many advances that have been made in this field include the advent of novel molecular-targeted therapies for lung cancer, the widespread use of inhaled corticosteroids, anti-cholinergic agents and long-acting beta-adrenergic agonists in the treatment of bronchial asthma/chronic obstructive pulmonary disease (COPD), and the development of novel antimicrobial agents for the treatment of respiratory tract infections. In regard to idiopathic pulmonary fibrosis, however, there have been no important additions to the armamentarium for managing this intractable disease over the last these 3 decades, since I graduated from medical school, with definitive therapeutics still lacking.

Lung tissue can be roughly divided into parenchyma and interstitium. In the narrower sense, the term "interstitium" refers to the alveolar septum which separates the alveoli from one another. Idiopathic interstitial pneumonias (IIPs) are characterized by hypertrophy of the alveolar septa due to inflammation. With progression of the disease, lung tissue remodeling through fibrosis takes place. According to the ATS/ERS International Multidisciplinary Consensus Classification (2013), IIPs can be classified into the following six major types: (1) idiopathic pulmonary fibrosis (IPF), (2) idiopathic nonspecific interstitial pneumonia, (3) respiratory bronchiolitis-interstitial lung disease, (4) desquamative interstitial pneumonia, (5) cryptogenic organizing pneumonia, and (6) acute interstitial pneumonia [1]. This classification is still not complete and is expected to be modified with advances in this research field.

IPF is the most frequent type of IIPs. Recent cohort studies and computed tomography (CT)-based epidemiological studies in Japan have indicated a high prevalence of IPF [2]. IPF is a disease characterized by intense lung fibrosis that follows a chronic and progressive course. In comparison to other types of IIPs, IPF responds poorly to steroid and immunosuppressant drug treatment and in general carries a poor prognosis. Approximately half of the patients with IPF die within $3-5$ years of the diagnosis. Acute exacerbations increase the mortality of IPF to as 
high as approximately $80 \%$. Thus, currently, IPF is among the most challenging of respiratory diseases to manage. Pulmonary fibrosis is also encountered in other conditions including viral infection, smoking, pneumoconiosis, medication, radiotherapy, and others. However, as indicated by its epithet "idiopathic," the causes and reasons for the lung fibrosis in IPF remain elusive. According to the current view, the essential feature of IPF is not inflammation caused by immunocompetent cells, but proliferation of fibroblasts (due to apoptosis of alveolar epithelial cells) and their differentiation into myofibroblasts. This view can explain why IPF responds poorly to treatment with steroids (glucocorticoids) (which have long been used clinically as representative anti-inflammatory agents). Furthermore, this view suggests the importance of anti-fibrotic therapy as an essential means of treatment for this condition.

Japanese researchers have made remarkable contributions to the study of this disease, some examples of which include the proposal of the concept of "acute exacerbation" [3], development of serum markers that are relatively highly specific for this disease (KL-6, SP-A, and SP-D) [4, 5], establishment of high-resolution computed tomography (HRCT) for diagnostic imaging, as compared to inflated and fixed lung specimens obtained by open lung biopsy and autopsy [6, 7], and reporting of the treatment outcomes of pirfenidone [8], an antifibrotic agent that was first approved in Japan. Thus, Japanese researchers have made remarkable contributions in the field of research on IIPs and published the results in international journals. Despite these diagnostic and therapeutic improvements, no treatments have been established yet that can boast of being a complete cure for IPF.

In addition, interstitial pneumonia with emphysema (combined pulmonary fibrosis and emphysema: CPFE) has been proposed recently as a new entity [9]. CPFE is a condition characterized by emphysematous lesions in the upper lung regions, accompanied by fibrosis in the lower lung regions. This disease is most often seen in male heavy smokers and is clinically characterized by the development of intense hypoxemia with effort, marked reduction in the lung dilatation, elevation of the serum KL-6 level, frequent complication by pulmonary hypertension and lung cancer, and so on. However, because of insufficient knowledge about the pathophysiology of this condition and the absence of a clear-cut definition, physicians in practice are still confused about this entity.

IPF is frequently complicated by lung cancer. It is therefore speculated that some common molecular mechanisms might be involved in the onset of IPF and lung cancer. The results of basic studies suggest that injury of the alveolar epithelial cells seen in cases of IPF not only stimulates pulmonary fibrosis, but also induces multiple gene abnormalities that can lead to the onset of lung cancer. It has also been suggested that apart from promoting lung carcinogenesis, pulmonary fibrosis may also be involved in the growth/spread of the cancer. Thus, basic research is under way in Japan to try to decode these associations. One of the important clinical problems associated with IPF is the development of acute exacerbations of the condition following surgery in lung cancer patients, but some very remarkable 
results of treatment are being obtained in large-scale epidemiologic studies in Japan [10].

To date, there have only been a small number of books containing detailed descriptions of IPF, all of which are uniformly organized in the traditional style. This current project presents readers with clinical questions about issues that are not yet fully resolved as the subtitles for the chapters, to authors who have long been engaged in research on IIPs long and are leading experts in this field in Japan. The authors will provide up-to-date information in response to each question. They will describe their thoughts and the future perspectives in response to the clinical questions, based on up-to-date information. Therefore, besides obtaining up-todate information, the readers can also understand the authors' real intentions and future perspectives, so that their intellectual curiosity will be satisfied.

Considering the nature of this project, useful information will be provided not only for beginning learners studying about IIPs, but also for physicians in clinical practice, instructors, and the many researchers engaged in basic research on this subject.

The Editors hope that this book, written by Japanese authors, will provide valuable input that will help all practicing physicians and medical researchers in the world to better understand the pathogenesis of IPF and to attempt to develop innovative treatments for this intractable disease. The Editors will be greatly pleased if this book can thus bring about therapeutic breakthroughs for patients worldwide suffering from IPF.

Ibaraki, Japan

Hiroyuki Nakamura

Kazutetsu Aoshiba

\section{References}

1. Travis WD, Costabel U, Hansell DM, King TE, Lynch DA, Nicholson AG, et al. An official American Thoracic Society/European Respiratory Society statement: Update of the international multidisciplinary classification of the idiopathic interstitial pneumonias. Am J Respir Crit Care Med. 2013;188(6):733-48.

2. Natsuizaka M, Chiba H, Kuronuma K, Otsuka M, Kudo K, Mori M, et al. Epidemiologic survey of Japanese patients with idiopathic pulmonary fibrosis and investigation of ethnic differences. Am J Respir Crit Care Med. 2014;190(7):773-9.

3. Kondoh Y, Taniguchi H, Kawabata Y, Yokoi T, Suzuki K, Takagi K. Acute exacerbation in idiopathic pulmonary fibrosis: analysis of clinical and pathologic findings in three cases. Chest. 1993;103(6):1808-12.

4. Kohno N, Kyoizumi S, Awaya Y, Fukuhara H, Yamakido M, Akiyama M. New serum indicator of interstitial pneumonitis activity: Sialylated carbohydrate antigen KL-6. Chest. 1989;96(1):68-73.

5. Honda Y, Kuroki Y, Matuura E, Nagae H, Takahashi H, Akino T, et al. Pulmonary surfactant protein D in sera and bronchoalveolar lavage fluids. Am J Respir Crit Care Med. 1995;152 (6 pt1):1860-6.

6. Nishimura K, Kitaichi M, Izumi T, Nagai S, Kanaoka M, Itoh H. Usual interstitial pneumonia: histologic correlation with high-resolution CT. Radiology. 1992;182(2):337-42. 
7. Johkoh T, Sakai F, Noma S, Akira M, Fujimoto K, Watadani T, et al. Honeycombing on CT; its definition, pathologic correlation, and future direction of its diagnosis. Eur $\mathrm{J}$ Radiol. 2014;83(1):27-31.

8. Azuma A, Nukiwa T, Tsuboi E, Suga M, Abe S, Nakata K, et al. Double-blind, placebocontrolled trial of pirfenidone in patients with idiopathic pulmonary fibrosis. Am J Respir Crit Care Med. 2005;171(9):1040-7.

9. Cottin V, Nunes H, Brillet PY, Delaval P, Devouassoux G, Tillie-Leblonde I, et al. Combined pulmonary fibrosis and emphysema: a distinct underrecognised entity. Eur Respir J. 2005;26 (4):586-93.

10. Sato T, Teramukai S, Kondo H, Watanabe A, Ebina M, Kishi K, et al. Impact and predictors of acute exacerbation of interstitial lung diseases after pulmonary resection for lung cancer. J Thorac Cardiovasc Surg. 2014;147(5):1604-11. 


\section{Contents}

Part I Definition, Epidemiology, and Pathogenesis

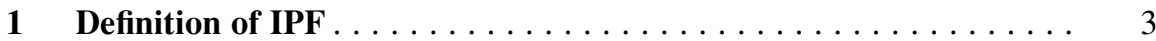

Shinji Abe and Akihiko Gemma

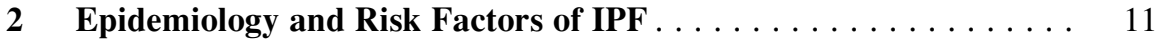

Kazuyoshi Kuwano, Jun Araya, and Hiromichi Hara

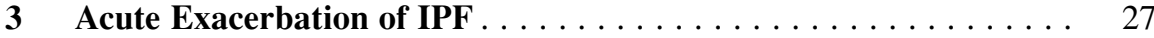

Yoshiki Ishii

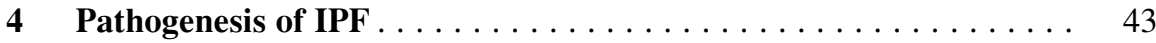

Yasuhiko Nishioka

\section{Part II Diagnosis}

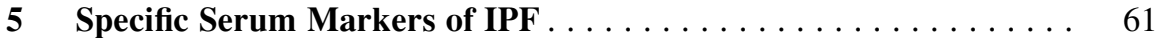

Hirofumi Chiba and Hiroki Takahashi

6 High-Resolution Computed Tomography of Honeycombing and IPF/UIP . . . . . . . . . . . . . . . . . . . . . . . . 77

Fumikazu Sakai

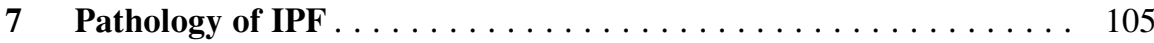

Yoshinori Kawabata

8 Differential Diagnosis of IPF . . . . . . . . . . . . . . . . . . . 133 Hidehiro Watanabe

Part III Management and Prognosis

9 Pharmacotherapy of IPF Using Antifibrotic Compounds . . . . . . . 147 Tomohiro Handa and Arata Azuma 
10 Pharmacotherapy of IPF (Corticosteroids, Immunosuppressants,

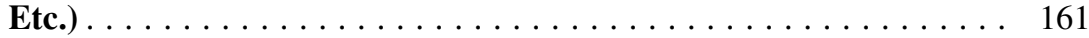
Masashi Bando

11 Non-pharmacological Therapy for IPF . . . . . . . . . . . . 171 Yukihiro Umeda, Tamotsu Ishizuka, and Takeshi Ishizaki

12 Pharmacotherapy of Acute Exacerbation of IPF (Corticosteroids, Immunosuppressants, and Direct Hemoperfusion with Polymyxin) . . . . . . . . . . . . . . . . . . . . . . . . 189 Masayuki Itoh

\section{Part IV Topics}

13 Combined Pulmonary Fibrosis and Emphysema (CPFE) . . . . . . . 205 Yoshiteru Morio and Kazuhisa Takahashi

14 Common Pathways in IPF and Lung Cancer . . . . . . . . . . . . . . 217 Nobuyuki Koyama

15 Acute Exacerbation of Interstitial Pneumonia After Pulmonary Resection for Lung Cancer . . . . . . . . . . . . . . . . . . . . . . . 249 Hiroshi Date 